\title{
EL SÍNDROME DE BURNOUT O DE QUEMARSE EN EL TRABAJO: UN CONFLICTO EN LA SALUD DE LOS PROFESIONALES.
}

\author{
BURNOUT SYNDROME OR BURNING AT WORK:
} A CONFLICT IN THE PROFESSIONALS' HEALTH.

\author{
Carolina Aranda B. ${ }^{1}$ \\ Instituto de Investigación en Salud Ocupacional Universidad de Guadalajara, México
}

(RECIBIDO EL 22/10 /2011, ACEPTADo EL 9/12/ 2011)

\begin{abstract}
RESUMEN
Diversos factores de riesgo derivados tanto de los medios de producción como de los modos de la organización pueden alterar la salud de los trabajadores o hasta la muerte. Precisamente una de las manifestaciones en la salud es el llamado Síndrome de burnout, mismo que es considerado como una fase avanzada del estrés laboral después de exponerse de manera continua al factor de riesgo. Su identificación, evaluación y control son imprescindibles para un adecuado desempeño laboral. El Síndrome de burnout es una enfermedad que trastorna la calidad de vida familiar, social y laboral de quien lo padece.
\end{abstract}

Palabras clave: Síndrome de burnout, riesgo, salud.

\begin{abstract}
Several risk factors derived from both the means of production and modes of organization can affect the health of workers or even death. Just one of the demonstrations in health is called burnout syndrome, which is considered as an advanced stage of work stress after being exposed continuously to the risk factor. Identification, evaluation and control are essential for adequate job performance. The burnout syndrome is a disease that disrupts the quality of family life, social and labor of whom suffers from it.
\end{abstract}

Keywords: burnout syndrome, risk, health. 


\section{INTRODUCCIÓN}

La frecuencia en el contacto y la cronicidad con los diversos factores de riesgo a los que están expuestos los trabajadores (profesionales de la salud, docentes a cualquier nivel de estudios y/o cualquier otra profesión), pueden ocasionar desde mínimas consecuencias en la salud y/o hasta la muerte.

Una de las consecuencias derivada de la presencia de factores de riesgo de tipo psicosocial que el trabajador percibe como negativos, es el Síndrome de burnout. A dicho síndrome se le considera como una fase avanzada del estrés laboral que puede llegar a causar incapacidad total para volver a trabajar o como una dolencia psíquica y hasta un accidente laboral, causante tanto de alteraciones en la salud (en ocasiones mortal) como de problemas en la organización.

El Síndrome de burnout también conocido como síndrome de quemarse o de desgaste profesional, se define entonces como un síndrome tridimensional con signos y síntomas característicos agrupados en tres dimensiones: agotamiento emocional, despersonalización y baja realización personal en el trabajo (Maslach y Jackson, 1981,1986).

Las cifras reportadas por la Organización Internacional del trabajo (OIT) sobre los accidentes y las enfermedades laborales a nivel general, demuestran que en el año 2007 se reportaban 2,2 millones anuales de trabajadores fallecidos por riesgos laborales a nivel mundial (Gómez, 2007). Más tarde, según reporta el periódico La Jornada en el 2009, había cinco mil quinientas muertes diarias a nivel mundial, en específico en el país México se reportan 2,9 por ciento de muertes por riesgos de trabajo por cada cien trabajadores (La Jornada, 2010).

\section{Factores de riesgo:}

Algunos de los factores de riesgo a los que los docentes están expuestos continúan siendo similares a los reportados en años anteriores, otros no, esto debido en algunos casos, a los avances tecnológicos, académicos, médicos, a los cambios sociales, la competitividad, etc., en el que este mundo globalizado se mueve.

Como factores de riesgo a nivel de docencia se observan el permanecer de pie, elevar y forzar la voz, jornadas extenuantes, recibir órdenes confusas, hacer guardias, permanecer en la institución en horas "muertas", supervisión estricta, el excesivo control de calidad, el revisar tareas, concentración excesiva, trabajo aburrido, descansos incómodos, posiciones incómodas, escribir en exceso en cuadernos y pizarrones (Rivero y Cruz, 2010) y, como factores de riesgo a nivel de los profesionales de la salud, en específico de los médicos se relacionan los siguientes: la edad, el sexo, el estado civil, la existencia o no de hijos, la antigüedad en el puesto, en la institución o en la empresa, la escolaridad, el ambiente físico de trabajo, experiencia laboral, jornadas extenuantes, turno laboral, carga horaria, actividad laboral excedida, interrelación entre los compañeros de trabajo y los superiores, jefes o directivos, falta o inadecuado apoyo social, sobreinvolucramiento, desarrollo de la carrera, uso de tecnologías nuevas, inseguridad, tipo de contratación, cambios en la organización, ser desempleado, 
falta de descansos, hasta el confort, entre otros (De las Cuevas, De la Fuente, Alviani y Ruíz, 1995; Tyler,1996; Gil Monte y Peiró,1997; Guevara,2004; Marqués,2005; Hernández,2006; Arias,2008; Camacho, Juárez y Arias, 2010; Castañeda y Garcia de Alba, 2010).

\section{Prevalencias:}

Las cifras de burnout reportadas para los docentes oscilan entre el $88 \%$ en maestros de preescolar (Galván, Aldrete, Preciado y Medina, 2010), hasta el $43 \%$ y un $80 \%$ en docentes de nivel primaria (Fernández, 2002, 2008: Aldrete, Pando, Aranda y Balcázar, 2003) respectivamente, mientras que a nivel de secundaria se reporta desde un 36,6\% (Fernández, 2008) al 80\% (Aldrete, González, Preciado y Pando, 2009). A nivel universitario los docentes presentan cifras de burnout del 68\% según (Aranda, Pando, Aldrete y Pérez, 2006), del 87,3\% (Paredes, 2001) y hasta del 94,7\% reportado por Bareño, Berbesi y Montoya (2010). Además, otros autores han estudiado el síndrome en escuelas privadas o particulares, reportando casi el $47 \%$ de burnout (Carlotto y Gongálves, 2004).

En cuanto a los porcentajes revelados sobre el síndrome de burnout en los médicos se perciben cifras que giran desde un $12 \%, 21,6 \%$ y $41,6 \%$ en médicos de atención primaria (Dickinson y cols, 2007; Matía y cols,2006; Castañeda y García de Alba, 2010) respectivamente. Así mismo se dan a conocer cifras del síndrome en profesionales de la salud que van desde el $31 \%$ (Ordenes, 2004) hasta un 36,6\% (Grau y cols, 2008) y un 42,2\% (Corredor y Monroy, 2009).

\section{Repercusiones en la salud:}

Una vez que se conocen las elevadas cifras de la presencia del síndrome de burnout, problema de salud pública bastante preocupante, es obvio darse cuenta que dicho padecimiento va más allá de ser un problema englobado en esas tres dimensiones de las que se habla, es decir, dicho síndrome a su vez originará otras repercusiones en la salud de las personas, en la sociedad, en su tarea laboral, en el grado de satisfacción con su trabajo y en su calidad de vida en general.

Son algunas de las manifestaciones en la salud tanto de los docentes como de los médicos relacionadas con el burnout la depresión, la paranoia, la desviación psicopática, la esquizofrenia, astenia, desajuste psicológico, el asma, los resfriados, diarreas, mareos, cefaleas, hipertensión, insomnio, poca apetencia, ansiedad, nauseas, úlceras, episodios depresivos, negación, desconexión mental y conductual, consumo de drogas, miedo al fracaso, creencias irracionales de perfeccionismo, alta necesidad de aprobación, baja autoestima, baja autonomía, poco control, fatiga, dolores musculares, trastornos vocales, respiratorios, pérdida de la memoria, trastornos del sueño, ausentismo laboral, evasión de responsabilidades, conflictos interpersonales, disminución de la productividad, desmotivación e insatisfacción laboral, preocupación, nerviosismo, irritabilidad, inquietud, distanciamiento, aumento de la crítica a la profesión y a la institución, trastornos digestivos, 
dermatitis, trastornos gastrointestinales, enfermedades crónicas en general, síntomas de estrés, etc.. (Aluja, 1997; Guerrero, 2001; Cordeiro, 2002; Guerrero, 2003; Rubio, 2003; Román, 2003; Martínez y cols, 2005; Diazgranado, González y Jaramillo, 2006; Latorre, 2006; García, Llorens, Cifre y Salanova, 2006; Rui Gomes y cols, 2006; Buendía, 2006; Aranda y cols, 2006b; Moreno y cols, 2006; Ortega, Ortiz y Coronel, 2007; Grau y cols, 2008; Grau y cols, 2009; Caballero y cols, 2009; Tejada y Gómez, 2009; Moreno, Garrosa y Rodríguez, 2009; Rivero y Cruz, 2010; Avargues, Borda y López, 2010; Velásquez, González y Contreras, 2010).

\section{Evaluación:}

Para fortuna, existen en la literatura diversos instrumentos que evalúan la presencia del síndrome de burnout tanto en los docentes como para los profesionales de la salud. A continuación se enlistan algunos instrumentos que ayudan en la evaluación del síndrome:

Los Instrumentos empleados para evaluar el síndrome en profesionales de la salud,:

1. La escala "Staff Burnout Scale for Health Professionals" (SBS-HP) realizado por Jones en 1980.

2. El "Maslach Burnout Inventory" (MBI) creado por Maslach y Jackson, 1981/1986.

3. El "SBS-HP" de Jones, elaborado en 1982.

4. EL "BM" creado por Pines y Aronson en 1988.

5. La escala "Efectos Psíquicos del Burnout" (EPB) creado por García y Velandrino, en 1992.

De otro lado, los Instrumentos empleados para evaluar el síndrome en profesionales de servicio de ayuda :

1. El "Burnout o Measure" (BM) de Pines y Aronson, 1988.

2. El "Maslach Burnout Inventory" (MBI) de Maslach y Jackson 1981/1986 .

3. El "Cuestionario Breve de Burnout" (CBB) por Moreno y Oliver en 1992.

4. El "Maslach Burnout Inventory" (MBI) por Maslach y Jackons en 1981/1986.

Los Instrumentos empleados para evaluar el síndrome en maestros y profesores :

1. El "Educators Survery" (ES) o "MBI forma Ed", adaptación del MBI por Schwab en 1986.

2. EL “Maslach Burnout Inventory" (MBI) creado por Maslach y Jackson en 1981/1986.

3. El "Cuestionario de Burnout del Profesorado" (CBP) creada por Moreno y Oliver en 1992.

4. El modelo de ecuaciones estructurales: el Structural Equation Modelling (SEM) por Llorens (2005).

5. El "Cuestionario de Burnout del Profesorado Revisado" (CBPR) por Moreno, Garrosa y González en el 2000.

6. El "Cuestionario Breve de Burnout" (CBB) adaptado para profesores por Moreno (1997). 
Entre los instrumentos más conocidos se encuentra la escala de valoración de Maslach Burnout Inventory (MBI) (Maslach y Jackson, 1981,1986). Dicho instrumento fue elaborado para profesionales de la salud, pero por su trascendencia ha sido empleado también (con sus respectivas adaptaciones) en los profesionales dedicados a la docencia.

Originalmente este instrumento contenía 25 ítems (Maslach y Jackson,1981,1986). Más tarde la misma escala queda con 22 ítems distribuidos en tres factores o subescalas, las cuales componen las tres dimensiones ya mencionadas (ver anexo 1):

1. Agotamiento emocional (Emotional exhaustion) (EE): describe "sentimientos de estar abrumado y agotado emocionalmente por el trabajo".

2. Despersonalización (Despersonalization) (D): "respuesta impersonal y falta de sentimientos hacia los sujetos objeto de atención” y,

3. Realización personal en el trabajo (Personal accomplishment) (PA): que comprende "sentimientos de competencia y realización en el trabajo y hacia los demás" (GilMonte y Peiró,1997).

El instrumento es valorado mediante una escala tipo Likert mediante 6 adjetivos que van desde "nunca" (0) hasta "todos los días" (6). Las puntuaciones obtenidas en la escala se colocan dentro una categoría o nivel de calificación o de identificación para cada de las dimensiones: nivel bajo, medio y alto. Se interpreta que una persona tiene "síndrome de burnout" cuando se encuentra en los niveles alto y medio, mientras que cuando lo está en los niveles bajos se le considerara como "no burnout".

\section{CONCLUSIONES}

El Síndrome de burnout, como se puede observar, es entonces una enfermedad "moderna", consecuente al estrés crónico de tipo laboral al que expuesto todo trabajador. Se dice síndrome ya que reúne una serie de signos y síntomas patológicos para ser considerado como tal. Las cifras sobre su presencia son realmente preocupantes y hasta alarmantes, sobre todo cuando se llega a la conclusión de que los profesionales aquí referidos, son quienes mantienen contacto directo con el paciente y con los alumnos, sin olvidar que también lo están con los familiares y con el resto de los compañeros de trabajo, desde los superiores hasta los subordinados, y que el síndrome ocasiona cambios de conducta, agresividad, problemas de relación con los compañeros de trabajo, con los hijos, familiares, etc. , así como probablemente una atención disminuida en potencial y motivación para realizar su actividad profesional. El Síndrome de burnout es una enfermedad que trastorna la calidad de vida familiar, social y laboral de quien lo padece.

Recordemos que el desempeño del personal de salud requiere de una serie de actividades que necesitan forzosamente de un control mental y emocional mucho mayor que en otros servicios e incluso en otras profesiones, sin embargo las cifras demuestran un alto porcentaje del síndrome en los docentes.

Es imprescindible identificar los factores causantes de este problema de salud, así como introducir medidas de prevención y/o de tratamiento a quienes lo requieran. 


\section{REFERENCIAS BIBLIOGRÁFICAS:}

ALDRETE, R.M.G., GONZÁLEZ, S.J., PRECIADO, S.M.L., PANDO, M.M. (2009). Variables sociodemográficas y el síndrome de burnout o de quemarse en profesores de enseñanza media básica (secundaria) de la Zona Metropolitana de Guadalajara. Revista de Educación y Desarrollo, 33-40.

ALDRETE, R.M.G., PANDO, M.M., ARANDA, B.C., BALCÁZAR, P.N. (2003). Síndrome de Burnout en maestros de educación básica, nivel primaria de Guadalajara. Investigación en salud, 5(1):11-16.

ALUJA, A. (1997). Burnout profesional en maestros y su relación con indicadores de salud mental. Boletín de Psicología, 55:47-61.

ARANDA, B.C., PANDO, M.M., TORRES, L.T.M., SALAZAR, E.J.G., ALDRETE, R.M.G. (2006b). Síndrome de burnout y manifestaciones clínicas en los médicos familiares que laboran en una institución de salud para los trabajadores del Estado. Psicología y Salud, 16(1):15-21.

ARANDA, B.C., PANDO, M.M., AldRETE, R.M.G., PÉREZ, R.M.B. (2006). La mujer docente y administrativa del Centro Universitario de Ciencias de la Salud (CUCS), México, y su relación con la presencia el Síndrome de Burnout. Revista de Educación y Desarrollo, 51.

ARIAS, F., BARBOZA, N., BLANCO, J., FAJARDO, E., RIVERA, R., RODRÍGUEZ, S., UGALDE, S., LÉPIZ, R. (2008). Síndrome del desgaste profesional o de burnout en el personal de enfermería. Revista Enfermería, 13:1-18.

AVARGUES, M.L., BORDA, M., LÓPEZ, A.M. (2010). El core of burnout y los síntomas de estrés en el personal de Universidad. Prevalencia e influencia de variables de carácter sociodemográfico y laboral. Boletín de Psicología, 99:89-101.

BAREÑO, S.J., BERBESI, F.D.Y., MONTOYA, Z.C.P. (2010). Factores asociados al síndrome de burnout en docentes de enfermería, Medellín-Colombia 2008. Investig. Andina, 12(21):36-48.

BUENDÍA, J. (2006). Riesgos psicosociales en la universidad. El síndrome de "burnout" y el mobbing”. Revista Técnica de Salud Laboral y Prevención, 5-19.

CABALlERO, D.C., GONZÁleZ, G.O., MERCADO, A.D., LLANOS, B.C., y cols. (2009). Prevalencia del síndrome de burnout y su correlación con factores psicosociales en docentes de una institución universitaria privada de la ciudad de Barranquilla. Psicogente, 12(21):142-157.

CAMACHO, Á.A., JUÁREZ, G.A., ARIAS, G.F. (2010). Síndrome de Burnout y Factores Asociados en Médicos. Ciencia \& Trabajo, 35:251-256.

CASTAÑEDA, A.E., GARCÍA DE ALBA, G.J.E. (2010). Prevalencia del síndrome de agotamiento profesional (burnout) en médicos familiares mexicanos: análisis de factores de riesgo. rev. colomb. psiquiatr, 39(1):67-84.

CARLOTTO, M.S., GONÇALVES, C.S. (2004). Análise fatorial do maslach burnout inventory (mbi) em uma amostra de professores de instituições particulares. Psicologia em Estudo, Maringá, 9(3):499-505. 
CORDEIRO, C.J.A., QUILLÉN, G.C.L., GALA, L.F. (2002). El síndrome de Burnout en el profesorado de la escuela primaria de la Bahía de Cádiz. Revista de Ciencias de la Educación, 18:119-132.

CORREDOR, P.M.E., MONROY, F.J.P. (2009). Descripción y comparación de patrones de conducta, estrés laboral y burnout en personal sanitario. Hacia promoc. Salud, 14(1):109-123.

DIAZGRANADOS, S., GONZÁLEZ, C., JARAMILLO, R. (2006). Aproximación a las problemáticas psicosociales y a los saberes y habilidades de los docentes del Distrito. Rev. Estud. Soc, 23:45-55.

DICKINSON, B.M.E., GONZÁLEZ, S.C., FERNÁNDEZ, O.M.A., PALOMEQUE, R.P., GONZÁLEZ, Q.E., HERNÁNDEZ, V.I. (2007). Burnout Syndrome among Mexican Primary Care Physicians. Archivos en Medicina Familiar, 9(2):75-79.

DE LAS CUEVAS, C., DE LA FUENTE, J.A., AlViAnI, M., RUIZ, A. (1995). Desgaste Profesional y clima laboral en Atención Primaria. Mapfre Medicina, 6:7-18.

FERNÁNDEZ, A.M. (2008). Burnout, Autoeficacia y Estrés en Maestros Peruanos: Tres Estudios Fácticos. Ciencia \& Trabajo, 10(30):120-125.

FERNÁNDEZ, M. (2002). Desgaste psíquico (burnout) en profesores de educación primaria de Lima metropolitana. Persona, 5:27-66.

GAlVÁN, S.M.G., ALDRETE, R.M.G., PRECIADO, S.L., MEDINA, A.S. (2010). Factores psicosociales y síndrome burnout en docentes de nivel preescolar de una zona escolar de Guadalajara, México. Revista de Educación y Desarrollo, 14:5-11.

GARCÍA, R.M., LlORENS, S., CIFRE, E., SALANOVA, M. (2006). Antecedentes afectivos de la auto-eficacia docente: un modelo de relaciones estructurales. Revista de Educación, 339:387-400.

GIL-MONTE, P.R., PEIRO, J.M. (1997). Desgaste Psíquico en el Trabajo: El Síndrome de Quemarse. Madrid, Editorial Síntesis.

GÓMEZ, M.C. (2007). OIT: mueren 2.2 millones por accidentes laborales. Comunicados en prensa, periódico La Jornada del 7 de abril de 2007. Recuperado en http://www. jornada.unam. $\mathrm{mx} / 2007 / 04 / 07 /$ index $\cdot$ php? section $=$ politica $\&$ article $=011 \mathrm{n} 1 \mathrm{pol}$. Último acceso 26/03/2008.

GRAU, A., FLICHTENTREI, D., SUÑER, R., FONT-MAYOLAS, S., PRATS, M., BRAGA, F. (2008). El burnout percibido o sensación de estar quemado en profesionales sanitarios: prevalencia y factores asociados. Información psicológica, (91-92): 64-79

GRAU, A., FLICHTENTREI, D., SUÑER, R., PRATS, M., BRAGA, F. (2009). Influencia de factores personales, profesionales y transnacionales en el síndrome de burnout en personal sanitario hispanoamericano y español (2007). Rev Esp Salud Pública, 83(2): 215-230.

GUERRERO, B.E. (2001). Una investigación con docentes universitarios sobre el afrontamiento del estrés laboral y el Síndrome del Quemado. Revista Iberoamericana de Educación: 1-22. 
GUERRERO, B.E. (2003). Análisis pormenorizado de los grados de burnout y técnicas de afrontamiento del estrés docente en profesorado universitario. Anales de Psicología, 19(1):145-158.

GUEVARA, C.A., HENAO, D.P., HERRERA, J.A. (2004). Síndrome de desgaste profesional en médicos internos y residentes. Hospital Universitario del Valle, Cali. Revista Colomb Méd, 35(4):173-178.

HERNÁNDEZ, J.R. (2006). El desencuentro entre la salud mental y la salud de los trabajadores. Rev Cubana Salud Pública, 32(4):106-110.

LA JORNADA. (2009). Mueren más mexicanos por males laborales que por epidemias: Cilas. Periódico La Jornada del 13 de mayo de 2009. Accesible en http: //www. jornada.unam. $\mathrm{mx} / 2009 / 05 / 13 /$ index.php? section $=$ socie dad. Último acceso 26/04/2011.

LA JORNADA. (2010). Aumentan accidentes laborales. Periódico La Jornada, 8 de febrero. Recuperado en: http://www.jornada.unam.mx/2010/02/08/index.php? section $=$ politica $\&$ article $=016 n 1$ pol Último acceso 14/02/2011.

LATORRE, R.I. (2006). Burnout en la enseñanza: análisis de una población de profesores de un colegio concertado de la Región de Murcia. Pedagogía Social. Revista interuniversitaria, 12-13:267-278.

MARQUÉS, A., LIMA, M.L., LOPES DA SILVA, A. (2005). Fuentes de estrés, burnout y estrategias de coping en profesores portugueses. Revista de Psicología del Trabajo $y$ de las Organizaciones, 21(1-2):125-143.

MARTÍNEZ, I.M., GRAU, R., LLORENS, S., CIFRE, E., y cols. (2005). Efectos del desajuste obstáculos-facilitadores organizacionales en el estrés docente: un estudio longitudinal. Revista Orientación Educacional, 35:1-20.

MASLACH, C., JACKSON, S.E. (1981). Maslach Burnout Inventory. Palo Alto California, Consulting Psychologists.

MASLACH, C., JACKSON, S.E. (1986). Maslach Burnout Inventory. 2da. Edición. Palo Alto California, Consulting Psychologists Press.

MATÍA, C.A.C., CORDERO, G.J., MEDiAVILlA, B.J., PEREDA R.M.J. (2006). Prevalencia de burnout y variables asociadas en los médicos de atención primaria de Burgos. Atención Prirmaria, 38(1):58-59.

MORENO, J.B., HERNÁNDEZ, G.E., RODRÍGUEZ, C.R., MARTÍNEZ, G.M., y cols. (2009). El burnout del profesorado universitario y las intenciones de abandono: un estudio multi-muestra. Revista de Psicología del Trabajo y de las Organizaciones, 25(2):149-163.

MORENO, J.B., MORETT, N.N.I., RODRÍGUEZ, M.A., MORANTE, B.M.E. (2006). La personalidad resistente como variable moduladora del síndrome de burnout en una muestra de bomberos. Psicothema,18(3): 413-418.

ORDENES, D.N. (2004). Prevalencia de Burnout en trabajadores del hospital Roberto del Río. Revista Chilena de Pediatría, 75(5):449-454. 
ORTEGA, H.M.E., ORTIZ, V.G.R., CORONEL, B.P.G. (2007). Burnout en médicos y enfermeras y su relación con el constructo de personalidad resistente. Psicología y Salud, 17(1):5-16.

PAREDES, S.M.C. (2001). Caracterización multivariante del síndrome de burnout en la plantilla docente de la universidad de Salamanca. Tesis de grado. Universidad de Salamanca de España.

RIVERO, R.L.F., CRUZ, F.M.A. (2010). Trastornos psíquicos y psicosomáticos: problemas actuales de salud de los docentes mexicanos. Psicología y Salud, 20(2):239249.

ROMÁN, H.J. (2003). Estrés y Burnout RODRIGUEZ, M. J. 1995. Psicología Social de la Salud. Madrid, Editorial Síntesis.

RUBIO, J.J.C. (2003). Fuentes de estrés, Sindrome de Burnout y Actitudes disfuncionales en Orientadores de Instituto de Enseñanza Secundaria. Tesis Doctoral. Universidad de Extremadura. Servicios de Publicaciones.

RUI, G.A., JOAO, S.M., MOURISCO, S., SILVA, S., MOTA, A. (2006). Problemas e desafios no exercício da actividade docente: Um estudo sobre o stresse, "burnout", saúde física e satisfação profissional em professores do $3^{\circ}$ ciclo e ensino secundario. Revista Portuguesa de Educação, 19(1): 67-93.

TYLER, M., ABBOTT, P. (1994). The commodification of sexuality: Sexuality labour markets. En Relación entre género y envejecimiento. Madrid, Editorial Síntesis, 1996.

TEJADA, M.P.A., GÓMEZ, O.V. (2009). Psychosocial and Work Factors Associated with Burnout in Colombian Psychiatrists. Rev. Colomb. Psiquiatr, 38(3):488-512.

VELÁSQUEZ, P.L., GONZÁleZ, G.M., CONTRERAS, G.A. (2010). Síntomas Depresivos y Agotamiento en Personal Trabajador del Instituto Nacional de Neurología y Neurocirugía. Revista Argentina de Ciencias del Comportamiento, 2(2): 60-67. 


\section{ANEXO 1 \\ ESCALA DEL MASLACH BURNOUT INVENTORY (MBI)}

Coloque en el cuadro vacío el número que corresponda según la opción que escoja. $0=$ Nunca $1=$ Alguna vez al año o menos $2=$ Una vez al mes o menos $4=$ Una vez por semana $5=$ Algunas veces por semana 6 : Todos los días

\begin{tabular}{|l|l|}
\hline \multicolumn{1}{|c|}{ ITEMS } & $\begin{array}{c}\text { Coloque el } \\
\text { número según la } \\
\text { opción elegida }\end{array}$ \\
\hline 1EE $\quad$ Debido a mi trabajo me siento emocionalmente agotado. & \\
\hline 2EE $\quad$ Al final de la jornada me siento agotado & \\
\hline 3EE $\quad$ Me encuentro cansado cuando me levanto por las mañanas y tengo que & \\
\hline 4PA $\quad$ Puedo entender con facilidad lo que piensan mis pacientes. & \\
\hline 5D $\quad$ Creo que trato a algunos pacientes como si fueran objetos. & \\
\hline 6EE $\quad$ Trabajar con pacientes todos los días es una tensión para mí. & \\
\hline 7PA $\quad$ Me enfrento muy bien con los problemas que me presentan mis pacientes. & \\
\hline 8EE $\quad$ Me siento "quemado" "fatigado" por el trabajo. & \\
\hline 9PA $\quad$ Siento que mediante mi trabajo estoy influyendo positivamente en la vida de \\
otros.
\end{tabular}

